\title{
Imaging and identification of point defects in $\mathrm{PtTe}_{2}$
}

\author{
Kuanysh Zhussupbekov $\mathbb{D}^{1,2,6}$, Lida Ansari $\mathbb{D}^{3,6}$, John B. McManus ${ }^{2,4}$, Ainur Zhussupbekova $\mathbb{D}^{1,2}$, Igor V. Shvets ${ }^{1,2}$, \\ Georg S. Duesberg $\mathbb{1}^{4,5}$, Paul K. Hurley ${ }^{2,3}$, Farzan Gity $\mathbb{1}^{3 凶}$, Cormac Ó Coileáin ${ }^{2,4}$ and Niall McEvoy $\left(\mathbb{D}^{2,4 凶}\right.$
}

The properties and performance of two-dimensional (2D) materials can be greatly affected by point defects. PtTe 2 a $2 \mathrm{D}$ material that belongs to the group 10 transition metal dichalcogenides, is a type-II Dirac semimetal, which has gained a lot of attention recently due to its potential for applications in catalysis, photonics, and spintronics. Here, we provide an experimental and theoretical investigation of point defects on and near the surface of $\mathrm{PtTe}_{2}$. Using scanning tunneling microscopy and scanning tunneling spectroscopy (STS) measurements, in combination with first-principle calculations, we identify and characterize five common surface and subsurface point defects. The influence of these defects on the electronic structure of $\mathrm{PtTe}_{2}$ is explored in detail through grid STS measurements and complementary density functional theory calculations. We believe these findings will be of significance to future efforts to engineer point defects in PtTe 2 , which is an interesting and enticing approach to tune the chargecarrier mobility and electron-hole recombination rates, as well as the site reactivity for catalysis.

npj 2D Materials and Applications (2021)5:14; https://doi.org/10.1038/s41699-020-00196-8

\section{INTRODUCTION}

The discovery of graphene ${ }^{1}$ has led to increasing interest in twodimensional (2D) materials for both fundamental research ${ }^{2-4}$ and hands-on applications ${ }^{5,6}$. 2D materials have a wide range of interesting properties ${ }^{7}$, that are not always achievable or apparent in their bulk form ${ }^{8}$. Thus, it has been recognized that 2D materials have the potential to become key players in the fields of energy ${ }^{9}$, electronics $^{5}$, and catalysis ${ }^{10,11}$, to name but a few. The extraordinary sensitivity of $2 \mathrm{D}$ materials can be influenced by a single defect or adsorbed molecule ${ }^{12}$. As $2 \mathrm{D}$ materials have moved toward the forefront of materials science research, obtaining a better understanding of featured defects, and their respective impacts, has become ever more important. Recently, to expand the available selection of 2D materials, there has been a concerted effort to synthesize and study new candidate materials. One such material is $\mathrm{PtTe}_{2}$ which is a member of the transition metal dichalcogenide (TMD) family $8,13,14$. While $\mathrm{PtTe}_{2}$ has thus far been less heavily studied than some other members of the TMD family, such as $\operatorname{MoS}_{2}{ }^{15-17}$, it benefits from interesting properties. It has been shown to be a type-II Dirac semimetal ${ }^{18}$ with high conductivity $^{19}$, suggesting it may be suitable for applications in spintronics and quantum computing ${ }^{20-22}$. Recently, the potential use of $\mathrm{PtTe}_{2}$ in a variety of different applications, such as electrocatalysis $^{11,23,24}$, ultrafast photonics ${ }^{25}$, and terahertz photodetection ${ }^{26}$ has been reported.

Defects on and near the surfaces of materials play a significant role in the electronic and atomic structure of the surface ${ }^{27}$. By controlling the concentration and types of defects, tunability of material properties is possible ${ }^{28}$. Furthermore, the presence of some quantity of zero-dimensional (OD) defects on or/and under the surface of a film is largely unavoidable, even under optimized synthesis conditions. These point defects can be interstitial impurities, vacancies, self-interstitial impurities, substitutional impurities, or a mixture of aforementioned defects. These OD defects induce surface states which can be either desirable or unwanted. The above-mentioned defects and impurities in thin films originate from the environment, the substrate, and synthesis conditions. Numerous recent studies have investigated the presence and influence of point defects in TMDs ${ }^{27,29-33}$.

Careful classification and characterization of these defects can allow us to understand their impact on the electronic properties of the intrinsic materials and assess their significance with a view to potential applications. The crystal structure of $\mathrm{PtTe}_{2}$ has been characterized by transmission electron microscopy (TEM) in several experimental studies ${ }^{19,21,34}$. Although TEM can routinely resolve the atomic structure of materials, it cannot provide easy access to the electronic band structure around the Fermi level of individual point defects. Moreover, recent reports have shown that TMDs are sensitive to electron beams, and distinguishing between native vacancy defects and those induced by the electron beam can be challenging ${ }^{35-37}$. Scanning tunneling microscopy and spectroscopy (STM/STS), on the other hand, can be used to probe the electronic structure of samples in a nondestructive manner ${ }^{38}$.

The ability to resolve atomic structure and point defects is a key attribute of STM. This is complemented by STS, which can be used to acquire information on the local density of states (LDOS). OD defects can exhibit altered minima or a maxima of electron density, which can manifest as depressions and protrusions, respectively, in STM images. A typical problem with detecting and classifying $0 D$ defects on the surface of a film arises from low areal density. Surface-sensitive techniques, such as X-ray photoelectron spectroscopy (XPS) and Raman spectroscopy, which are usually utilized in surface-compositional studies, are not sufficiently sensitive to distinguish these point defects. STM and STS are powerful and nondestructive techniques for the characterization of defects in materials; they are capable of atomic-scale resolution and can be used to locally probe the electronic properties of point defects.

The use of density functional theory (DFT) in conjunction with experimental STM images provides a path to establishing the atomic structure of the observed surface defects. In DFT-derived

\footnotetext{
${ }^{1}$ School of Physics, Trinity College Dublin, Dublin 2, Ireland. ${ }^{2}$ AMBER Centre, CRANN Institute, Trinity College Dublin, Dublin 2, Ireland. ${ }^{3}$ Nanoelectronics Materials and Devices, Tyndall National Institute, University College Cork, Cork, Ireland. ${ }^{4}$ School of Chemistry, Trinity College Dublin, Dublin 2, Ireland. ${ }^{5}$ Institute of Physics, EIT 2, Faculty of Electrical

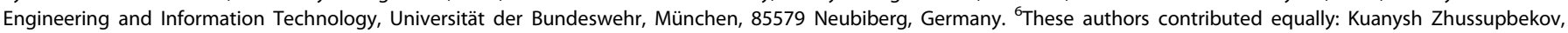
Lida Ansari. ${ }^{\circledR}$ email: farzan.gity@tyndall.ie; ocoilecl@tcd.ie; nmcevoy@tcd.ie
} 
STM images (DFT-STM), the tip is modeled as a locally spherical potential well and the tunneling current is proportional to the LDoS of the surface, at the position of the tip where it approaches nearest to the surface. It is assumed that the tip wave function has an s-orbital character and, at distances away from the surface, the partial density will be zero for localized basis set calculations since the basis orbitals have a finite range. DFT-STM images are sufficiently detailed and realistic to permit quantitative comparison with experimental STM images.

In this study, we image and identify five common distinct point defects on and near the $\mathrm{PtTe}_{2}$ surface. The $\mathrm{PtTe}_{2}$ samples examined in this work are synthesized through the reaction of solid-phase precursor films. Following an analysis of the $\mathrm{PtTe}_{2}$ samples with Raman spectroscopy, XPS, X-ray diffraction (XRD), and scanning electron microscopy (SEM), the $\mathrm{PtTe}_{2}$ surface is examined using STM and STS. The STM and STS experimental results are complemented and supported by DFT to probe, and understand the electronic properties of $\mathrm{PtTe}_{2}$, the characteristics of different defect types, and their impact on the electronic structure of $\mathrm{PtTe}_{2}$. These findings are a needed detailed examination of the local electronic properties of $\mathrm{PtTe}_{2}$, and are important in understanding the influence that point defects have on the material's properties and subsequent applications.

\section{RESULTS AND DISCUSSION}

$\mathrm{PtTe}_{2}$ film synthesis and characterization

Following the deposition of the Pt and Te layers on the substrate by sputtering and electrodeposition, respectively, the sample was annealed at $450^{\circ} \mathrm{C}$ for 90 min to synthesize the $\mathrm{PtTe}_{2}$ film ${ }^{11}$. XPS was employed to confirm the successful synthesis of $\mathrm{PtTe}_{2}$ films and to investigate their quality. Corresponding Pt $4 f$ and Te $3 d$ core-level regions of a film of $\mathrm{PtTe}_{2}$ are depicted in Supplementary Fig. 1a, b. Raman spectroscopy was also used to provide information on the quality of the material synthesized, as shown in Supplementary Fig. $1 c^{39}$. XRD allowed the crystallinity of the $\mathrm{PtTe}_{2}$ film to be examined (see Supplementary Fig. 1d). The sharp peaks observed are indicative of good crystallinity in the film, and are in agreement with previous reports ${ }^{11,18,40,41}$. SEM images of the $\mathrm{PtTe}_{2}$ film show its polycrystalline nature with grains ranging between 200 and $400 \mathrm{~nm}$ in size (see Supplementary Fig. 2). The combined measurements from these four complementary techniques confirm the successful synthesis of $\mathrm{PtTe}_{2}$ films.

\section{STM characterization of point defects}

During ex-situ transfer to ultrahigh vacuum (UHV) the sample was mounted on an STM puck. We performed a detailed STM investigation to study the atomic structure of the polycrystalline $\mathrm{PtTe}_{2}$ film, similar to previous studies focused on other materials ${ }^{29,42}$. The $1 \mathrm{~T}$ atomic structure of $\mathrm{PtTe}_{2}$ is schematically represented in Fig. 1a. Figure 1 shows STM images of a $\sim 20 \mathrm{~nm}$ thick $\mathrm{PtTe}_{2}$ film. Figure $1 \mathrm{~b}$, c present a large-area scan, different grains can be discerned and the majority of these have hexagonal forms, consistent with SEM observations. The noticeable surface contamination present likely originates from the sample being exposed to ambient conditions between synthesis and analysis. Figure $1 \mathrm{~d}$ is an atomic-resolution STM of a defect-free area of the $\mathrm{PtTe}_{2}$ surface (area indicated by the blue square in Fig. 1c), confirming the $1 \mathrm{~T}$ crystal structure of the $\mathrm{PtTe}_{2}$ grains. An average lattice constant and a layer step height for the $\mathrm{PtTe}_{2}$ of $4.0 \pm 0.03$ and $5.1 \pm 0.06 \AA$, respectively, were determined from the atomically resolved surface, which is in agreement with our simulated results and previous experimental reports ${ }^{40,41}$. Considering this, we estimate from STM that the thickness of the flakes in our measurements ranged from 30 to 50 layers.

Figure 1e, $f$ are STM images of the same area of the $\mathrm{PtTe}_{2}$ surface, allowing for drift, scanned at positive and negative biases, corresponding to empty and filled states, respectively, which demonstrate a variety of defect types. The contrasting defect appearance between Fig. $1 \mathrm{e}, \mathrm{f}$ is in response to the change of bias
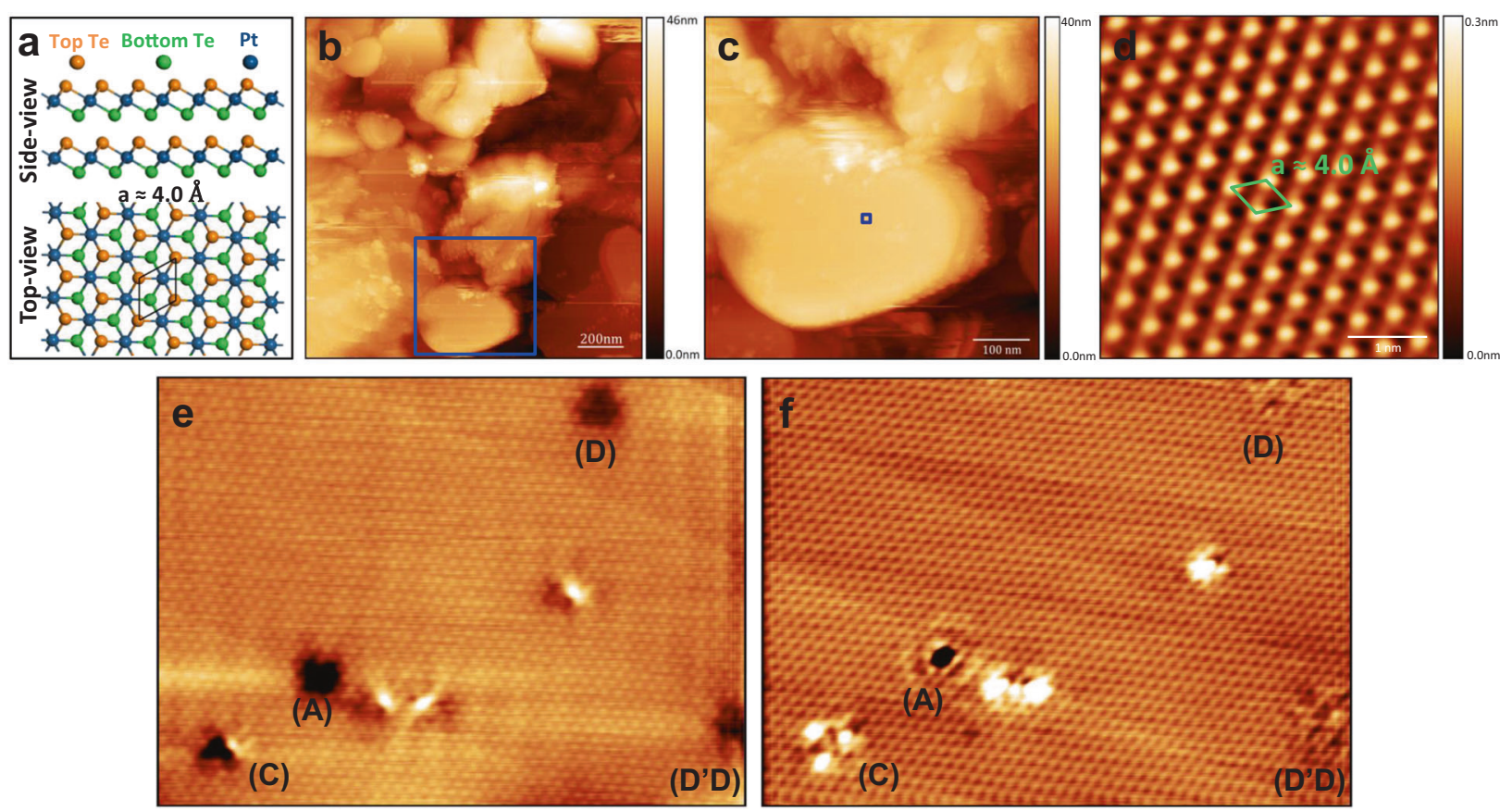

Fig. 1 STM images of the polycrystalline PtTe $\mathbf{P}_{2}$ film. a Schematic view of 1T-PtTe. b Large-scale STM image of the PtTe 2 polycrystalline film $(1500 \times 1500 \mathrm{~nm}, V=1.5 \mathrm{~V}$ and $I=80 \mathrm{pA})$. c STM image exhibits some contaminants present at the upper edge on the surface of the PtTe $e_{2}$ crystal $(600 \times 600 \mathrm{~nm}, V=1.5 \mathrm{~V}$ and $I=60 \mathrm{pA})$. d STM image showing atomic structure of $\mathrm{PtTe}_{2}$ in a defect-free area with an interatomic

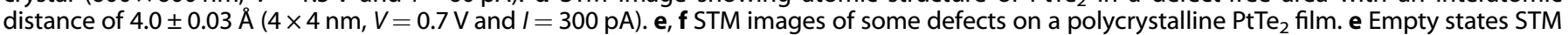
image of the $\mathrm{PtTe}_{2}$ film $(20 \times 15 \mathrm{~nm}, V=0.85 \mathrm{~V}$ and $I=185 \mathrm{pA})$. f Filled states STM image of the PtTe $e_{2}$ film $(20 \times 15 \mathrm{~nm}, V=-0.85 \mathrm{~V}$ and $I=$ $185 \mathrm{pA})$. 
from positive to negative, i.e., their brightness (contrast) changes. The clear distinction between the featured defects indicates different atomic configurations within each type. An additional STM image, in which four different defect types are visible, is included in Supplementary Fig. 3. By resolving each defect in their empty and filled states, we identified five types of point defects at the $\mathrm{PtTe}_{2}$ surface, which we label henceforth as A, B, C, D, and E. We noticed a trend where defects with a bigger presence in terms of size on the surface appeared less bright, modeling correlated this with defect depth from the surface, similar to previous reports on $\mathrm{PtSe}_{2}{ }^{29}$.

Figure $2 a-e^{\prime}$ are high-magnification STM images of the five defect types at empty and filled states. Defects of type A appear as depressions at both positive and negative bias voltages. Defects of types B, C, D, and E display protrusions (depressions) at negative (positive) bias voltages. Defects of types B, C, and E have clear threefold symmetry. While defect type $D$ has a sixfold symmetry at positive bias (empty states) and threefold symmetry at negative bias (filled states). Table 1 displays a rough estimation of the defect density, based on cumulative counts within areas imaged with atomic resolution before $\left(\sim 1000 \mathrm{~nm}^{2}\right)$ and after $\left(\sim 2200 \mathrm{~nm}^{2}\right)$ annealing. While the sampled areas were relatively small, and limited to areas of minimal contamination, some interesting trends are apparent. Among these five types, tellurium vacancies, defects of types $A$ and $C$ are the most common at the $\mathrm{PtTe}_{2}$ surface, which is consistent with the calculated defect formation energy. Furthermore, the density of these defects diminishes most readily after annealing at $430^{\circ} \mathrm{C}$ for $3 \mathrm{~h}$ in UHV. However, while several flakes were examined before and after annealing, a distribution in defect density could be expected due to the polycrystalline nature of the film and the defect density will inherently depend on the synthesis conditions. We shall now discuss each defect type separately.

Examining the defects on an individual basis, type A defects appear as depressions on the surface at both bias polarities (see Fig. 2a). However, there is a small difference in the brightness of the STM images surrounding the depression for negative bias voltages (filled states) that confers it a crown shape. It is thought that this type of defect is a Te vacancy in the top Te layer of the surface of $\mathrm{PtTe}_{2}$ layer.

Type $B$ defects are of a larger $6 \times 6$ triangular depression shape at positive bias voltages. However, at negative bias voltage the defect is characterized by a small protrusion of the same triangular area (see Fig. 2b). This is the largest among the observed defect types, and the large lateral dimensions suggest that the defect is positioned deeper than the rest of the defects ${ }^{29}$.

Defects of type $C$ look like a lobed $3 \times 3$ triangular depression at positive bias voltage and a small depressed triangle flanked by a triad of distinctive protruding spots at negative bias voltage (see Fig. 2c). Tunneling currents tend to change in response to atomic positions and differences in LDoS. Typically, protrusions in STM are the result of a higher LDoS. The LDoS of this type of defect will be discussed in further detail in the STS characterization section.

Type $D$ defects resemble a hexagonal depression at positive bias (see Fig. 2d). However, scanning at negative bias voltages completely changes the appearance of the defect. There are three
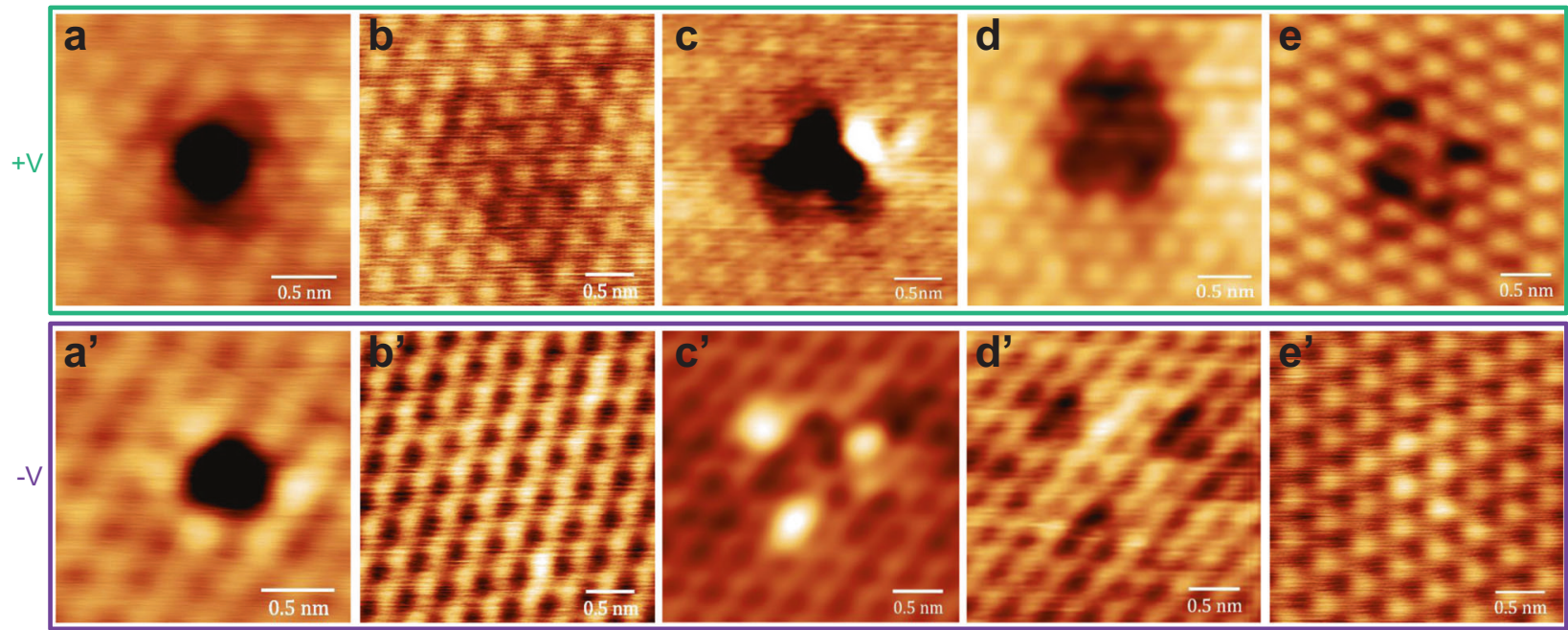

Fig. 2 Atomically resolved STM images of five types of point defects A to E scanned under positive and negative bias voltages. Top panel: STM images (a-e) of defects scanned under positive bias (empty states). Bottom panel: STM images ( $\left.\mathbf{a}^{\prime}-\mathbf{e}^{\prime}\right)$ of the same defects scanned under negative bias (filled states). Scanning parameters are: for type $A$ defect $(V= \pm 0.55 \mathrm{~V}, I=580 \mathrm{pA})$. Type $\mathrm{B}$ defect $(V= \pm 0.85 \mathrm{~V}, I=175 \mathrm{pA})$. Type $\mathrm{C}$ defect $(V= \pm 0.85 \mathrm{~V}, I=185 \mathrm{pA})$. Type $D$ defect $(V= \pm 0.85 \mathrm{~V}, I=185 \mathrm{pA})$. Type $\mathrm{E}$ defect $(V= \pm 0.9 \mathrm{~V}, I=250 \mathrm{pA})$.

Table 1. Densities of defects measured by STM.

\begin{tabular}{|llll}
\hline Defect type & Defect assignment & $\begin{array}{l}\text { Density of the defects observed } \\
\text { by STM as received }\left(1 \times 10^{11} \mathrm{~cm}^{-2}\right)\end{array}$ & $\begin{array}{l}\text { Density of the defects observed by STM after } \\
\text { annealing at } 430^{\circ} \mathrm{C} \text { for } 3 \mathrm{~h} \mathrm{in} \mathrm{UHV}\left(1 \times 10^{11} \mathrm{~cm}^{-2}\right)\end{array}$ \\
\hline A & Te vacancy in the top row & 8.1 & 1.4 \\
B & Pt vacancy in the & 6.0 & 0.5 \\
& second layer & & 1.8 \\
C & Te vacancy in the bottom row & 10.1 & 1.4 \\
D & Pt vacancy in the first layer & 2.0 & 0.9 \\
E & Antisite defect with Te & 1.0 & \\
\hline
\end{tabular}


small inward-pointing depressed triangles surrounding a slightly elevated central point. The shape of the defect scanned under negative bias looks like a trefoil. The transformation of this type of defect is interesting due to its complete change in a shape from sixfold symmetry to threefold symmetry when the bias polarity is changed.

Defect type $E$ is a $3 \times 3$ triangular depression at positive bias voltage (see Fig. 2e). However, for the STM image obtained at negative bias, the defect appears as a $3 \times 3$ triangular protrusion of the same shape as at positive bias but inverted. STS measurements on this type of defect are also discussed further.

Using STM, we can achieve atomic-resolution imaging of polycrystalline $\mathrm{PtTe}_{2}$ films. Furthermore, atomically resolved defects within the crystal lattice can be identified and distinguished by imaging their contrasting features with positive and negative biases. Additional examples of the defects we established are presented in the STM images in Supplementary Fig. 4.

STS characterization of defect-free $\mathrm{PtTe}_{2}$ surface and selected defects

To understand the electronic nature of the observed point defects, STS was used to investigate both pristine $\mathrm{PtTe}_{2}$ and defective sites. The STM/STS investigation of the point defects reveals their influence on the electronic properties, i.e., LDoS.

Figure 3a-d shows a grid STS investigation of the type $C$ defect in $\mathrm{PtTe}_{2}$. Figure 3a shows an STM image $\left(3 \times 3 \mathrm{~nm}^{2}\right)$ of the type $\mathrm{C}$ defect in $\mathrm{PtTe}_{2}$ and grid spectroscopy ${ }^{43}$ measurements performed on the same area (depicted in Fig. 3b). The grid was composed of $25 \times 25$ individual $I(V)$ measurements with dimensions of $3 \times$ $3 \mathrm{~nm}^{2}$. At each point the spectra were averaged over three individual $I(V)$ curves. The constant current mode (CCM) scanning parameters to move the tip between points were $V=0.6(\mathrm{~V})$ and $I$ $=740(\mathrm{pA})$. Variation in the tunneling current at different voltages is due to differences in the electronic structure. The voltage was swept between +1 and $-1 \mathrm{~V}$. Figure $3 \mathrm{~b}$ depicts the tunneling current from the $I(V)$ at each spectroscopy point for a bias voltage value of $-0.150 \mathrm{~V}$, which corresponds to the filled states. While Fig. $3 d$ shows the tunneling current from the $I(V)$ at each spectroscopy point for a bias voltage value of $+0.148 \mathrm{~V}$, which corresponds to the empty states. Darker/brighter points in Fig. 3b, $\mathrm{d}$ correspond to a lower/higher tunneling current at $-0.150 \mathrm{~V}$ (filled states) and $+0.148 \mathrm{~V}$ (empty states), respectively. The blue (point 1) and red (point 2) stars in Fig. 3a mark points on the defect-free terrace and the type $C$ defect, respectively. These regions are also marked in Fig. $3 \mathrm{~b}, \mathrm{~d}$. It is interesting to note that the defect edge exhibits a higher LDoS compared to the flat defect-free terrace. These altered electronic properties in the vicinity of the defect can be understood in terms of a Te vacancy that would create a high acceptor density resulting in the triangular shape, which gives rise to the three protrusions seen in Figs $3 \mathrm{~d}$ and $2 \mathrm{c}^{\prime}$. Figure $3 \mathrm{c}$ displays the comparative $I(V)$ and $\mathrm{d} / \mathrm{d} V$ (numerical derivative of $I(V)$ ) spectra of the two marked points. From Fig. $3 c$, it can be seen that these three dark/bright points on the defect have a much higher LDoS in the filled states in comparison with the empty states. The LDoS of the defect is boosted to almost twice that of the pristine terrace in the filled states. However, the LDoS of the defect in the empty states only changes slightly compared to that of the pristine terrace, except in the region from 0.4 to $0.77 \mathrm{~V}$ where it is slightly diminished. This suggests that it is probably a $p$-type defect.

Figure $3 \mathrm{e}-\mathrm{h}$ detail grid STS analysis of the type $\mathrm{E}$ defect in $\mathrm{PtTe}_{2}$. An STM image of a type $E$ defect is shown in Fig. $3 e\left(4 \times 4 \mathrm{~nm}^{2}\right.$, $V=0.9 \mathrm{~V}$ and $I=250 \mathrm{pA}$ ). Grid spectroscopy measurements in Fig. $3 f$ were performed on the same area. The $4 \times 4 \mathrm{~nm}^{2}$ grid is composed of $30 \times 30$ measurements each averaged over three individual $I(V)$ curves. The voltage was swept from -2.2 to $+1.7 \mathrm{~V}$. Figure $3 f$ depicts the tunneling current from the $I(V)$ at each spectroscopy point at a bias value of $-2.2 \mathrm{~V}$, which corresponds to the filled states. While, Fig. 3h shows the tunneling current from
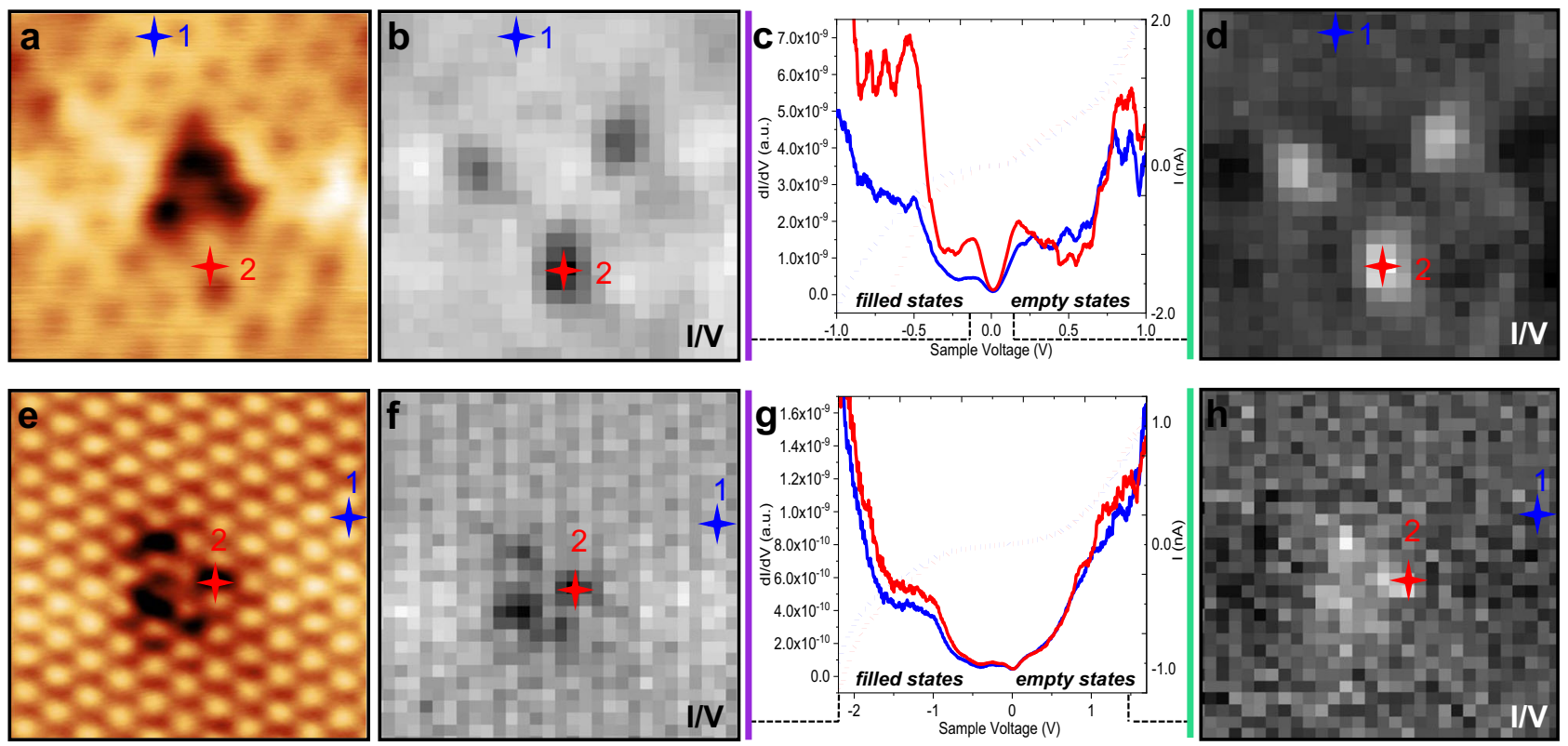

Fig. 3 Grid spectroscopy of PtTe ${ }_{2}$ type $C$ and E defects. a STM topography of the type $C$ defect $\left(3 \times 3 \mathrm{~nm}^{2}, V=0.6 \mathrm{~V}\right.$ and $\left.I=740 \mathrm{pA}\right)$. Grid spectroscopy was performed on the area in $\mathbf{a}$. $\mathbf{b} I / V$ map depicts the tunneling current value (taken from the $I(V)$ ) at each spectroscopy point on the grid $(25 \times 25$ points) at a bias voltage value of $-0.150 \mathrm{~V}$ (filled states). Three dark points correspond to lower tunneling current of the defect on the filled states in PtTe 2 . c Individual I/V (dotted line) and d//dV (solid line) spectra of two points indicated by the colored stars in a, $\mathbf{b}$, and $\mathbf{d}$ positioned on a flat terrace and on the defect. $\mathbf{d} I / \mathrm{V}$ map at $+0.148 \mathrm{~V}$ above the Fermi level. e STM topography of the type $\mathrm{E}$ defect $(4 \times$ $4 \mathrm{~nm}^{2}, V=0.9 \mathrm{~V}$ and $I=250 \mathrm{pA}$ ). Grid spectroscopy was performed on the area in e. f $I / V$ map depicts the tunneling current value (taken from the $I(V)$ at each spectroscopy point on the grid $(30 \times 30$ points) at a bias voltage value of $-2.2 \mathrm{~V}$. g Individual $I / V$ (dotted line) and d $/ / \mathrm{d} V$ (solid line) spectra of two points on the grid indicated by the colored stars in $\mathbf{e}, \mathbf{f}$, and $\mathbf{h}$ positioned on a defect-free terrace and on the defect. $\mathbf{h} I / V$ map at $+1.48 \mathrm{~V}$ above the Fermi level. 
the $I(V)$ at each spectroscopy point for a value of $+1.48 \mathrm{~V}$ above the Fermi level, which corresponds to the empty states. It should be noted that the defect has a slightly higher LDoS compared to the pristine surface of $\mathrm{PtTe}_{2}{ }^{29,44}$. Figure $3 \mathrm{~g}$ illustrates $I(V)$ and $\mathrm{d} / / \mathrm{d} V$ spectra of two points on the grid indicated by the colored stars positioned on the pristine surface (blue point 1 ) and on the type $E$ defect (red point 2). From Fig. 3g, it can be seen that the dark side (defect has dark contrast at $-2.2 \mathrm{~V}$ and bright contrast at $+1.48 \mathrm{~V}$ ) of the defect has a slightly higher LDoS in the filled states in comparison with the LDoS of the pristine surface. The LDoS of the defect in the empty states fluctuates over the $d / / d V$ spectra of the pristine surface. Due to the challenging nature of grid STS, only two types of defects are shown here. Point STS acquired from defect type $A$ is presented in Supplementary Fig. 5.

Electronic structure of $\mathrm{PtTe}_{2}$

Ab-initio techniques were applied to study the electronic properties of $\mathrm{PtTe}_{2}$ in the forms of ultrathin films and bulk. Figure 4 illustrates the side view schematic and the band structure of $\mathrm{PtTe}_{2}$. This figure compares the band structures of pristine monolayer $(\mathrm{ML})$, bilayer $(\mathrm{BL})$, and bulk $\mathrm{PtTe}_{2}$ obtained by generalized gradient approximation (GGA) and GGA-1/2, considering spin-orbit coupling (SOC). Band structures when the SOC is not accounted for are shown in Supplementary Fig. 6. As can be seen in Fig. 4b, $c$, the $\mathrm{ML} \mathrm{PtTe}_{2}$ exhibits an indirect bandgap of $0.37 \mathrm{eV}$ for the GGAobtained band structure. The indirect bandgap increases to $0.9 \mathrm{eV}$ for the GGA-1/2 technique, which is comparable to the bandgap of conventional semiconductors, making $\mathrm{ML} \mathrm{PtTe}{ }_{2}$ of interest for electronic applications, such as field effect transistors, where the influence of dopants is critical. As indicated by the red arrows in Fig. 4c, GGA-1/2 has reduced and "flattened" the curvature of the valence bands, as well as shifting the conduction band (valence band) up (down) in energy; hence, the increased bandgap. In the $\mathrm{ML} \mathrm{PtTe} \mathrm{P}_{2}$ band structure, the valence band maximum (VBM) is at the $\Gamma$ point and there are two competing conduction band minima (CBM) at either side of the $\Gamma$ point. The CBM obtained by GGA is between the $\Gamma$ and $\mathrm{K}$ points $(\triangle E \approx 30 \mathrm{meV})$, while for the GGA-1/2 the CBM is between the $\Gamma$ and $M$ points $(\Delta E \approx 25 \mathrm{meV})$.

When the thickness of $\mathrm{PtTe}_{2}$ increases from $\mathrm{ML}$ to $\mathrm{BL}$, a similar double CBM is observed, as can be seen in Fig. 4e, f. Also, the VBM splits into two peaks centered at the $\Gamma$ point, with a similar bandgap widening and flattening effect of the GGA-1/2 approach. Nevertheless, the energy level of the VBM exceeds that of the
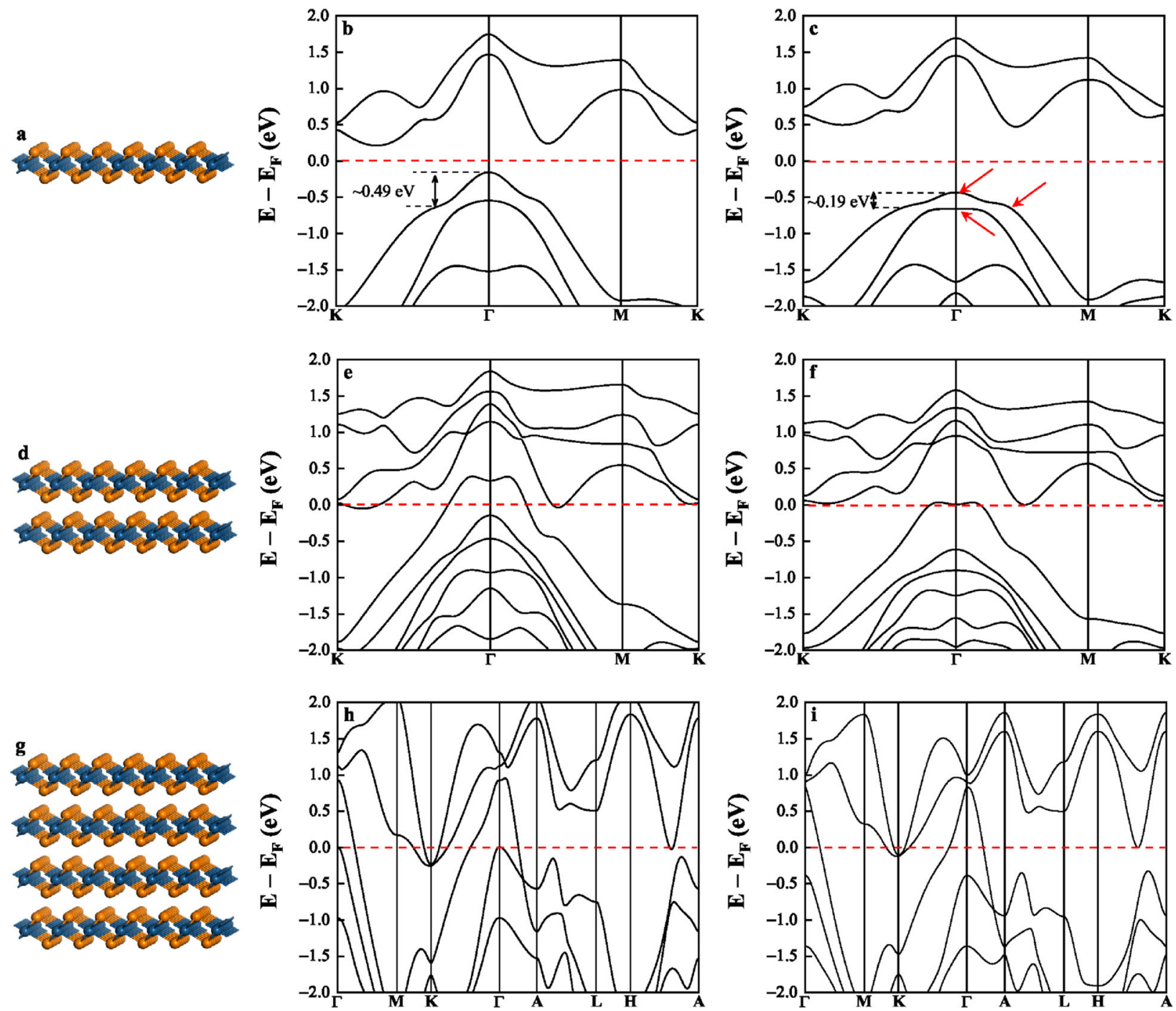

Fig. 4 Atomic and electronic structure of pristine PtTe $\mathbf{e}_{2}$. Side view schematic (left column: $\mathbf{a}$, $\mathbf{d}$, and $\mathbf{g}$ ), and band structure considering GGA + SOC (middle column: b, e, and $\mathbf{h}$ ) and GGA-1/2 + SOC (right column: $\mathbf{c}, \mathbf{f}$, and i), of monolayer PtTe 2 (top row), bilayer PtTe 2 (middle row), and bulk $\mathrm{PtTe}_{2}$ (bottom row). Modification of the band structure is pronounced when the GGA-1/2 technique is employed. Transition from semiconductingto-semimetallic is evident by the closure of bandgap as the film thickness increases. Energies are referenced to the Fermi energy. 
CBM, even using the GGA-1/2 technique, leading to an overlap of bands; hence, a semimetal-to-semiconductor transition occurs in this material due to quantum confinement. This is consistent with angle-resolved photoemission studies that have shown BL PtTe to be metallic ${ }^{34}$. The band overlap increases even further for bulk $\mathrm{PtTe}_{2}$ as shown in Fig. 4h, i. Such a phenomenon has also been reported in literature for some other semimetal materials, e.g., Sn, $\mathrm{Bi}$, and $\mathrm{PtSe}{ }_{2}{ }^{45-48}$. Orbital-resolved and atom-resolved DoS for $\mathrm{ML}$, $\mathrm{BL}$, and bulk $\mathrm{PtTe}_{2}$ are presented in Supplementary Fig. 7. It can be observed that for $\mathrm{ML}, \mathrm{BL}$ and bulk $\mathrm{PtTe}_{2}$ the $p$ - and $d$-orbitals contribute almost equally to the conduction band states, while the valence band states dominantly originate from the $p$-orbitals compared to $d$-orbitals, with more than ten times less contribution from s-orbitals. These results also imply that the contribution of Te atoms in the DoS is similar to that of Pt atoms. A hybrid functional, based on a screened Coulomb potential included in the exchange functional of plane-wave based DFT, has been shown to avert the bandgap underestimation in DFT calculations ${ }^{49}$. To investigate the bandgap correction with GGA-1/2 method for $\mathrm{PtTe}_{2}$, we have also considered the hybrid functional for $\mathrm{ML} \mathrm{PtTe}_{2}$, results of which are shown in Supplementary Fig. 8. The value of the bandgap and the band profile of the GGA-1/2 method are in very good agreement with the hybrid functional, as can be seen in the figure.

To gain more insight into the characteristics of the defects and their impact on the electronic structure of $\mathrm{PtTe}_{2}$, in particular on the vacancy-induced energy states and the location of the Fermi level in the bandgap, we systemically explored the properties of both $\mathrm{Pt}$ and $\mathrm{Te}$ neutral vacancies in $\mathrm{PtTe}_{2}$ using first-principle calculations. According to Fig. 4, only $\mathrm{ML} \mathrm{PtTe} 2$ exhibits a bandgap; hence, $\mathrm{ML} \mathrm{PtTe} 2$ has been considered in this part of the study.

Figure 5 shows the band structure of $\mathrm{ML} \mathrm{PtTe}_{2}$ with a single Te vacancy (parts a and $b$ ) and single Pt vacancy (parts $c$ and $d$ ) obtained from GGA + SOC and GGA-1/2 + SOC approaches. For the Te vacancy, as can conspicuously be seen, defective bands emerge in both the upper and lower half of the bandgap compared to the band structure of pristine $\mathrm{ML} \mathrm{PtTe}$ (Fig. 4, top row). Note that the energies are referenced to the Fermi energy and for the Te vacancy, the Fermi level is almost at the middle of the bandgap for all calculation approaches. For the Pt vacancy on the other hand, the defect-induced energy states in the bandgap are located in the lower half of the bandgap and the Fermi energy is closer to the VBM; i.e., presenting a $p$-type characteristic. GGAand GGA-1/2-derived band structures (without SOC) of ML PtTe with $\mathrm{Te}$ and Pt vacancies are presented in Supplementary Fig. 9, where spin degeneracy in the defect states can be observed. Comparing Fig. 5 and Supplementary Fig. 9, large spin-orbit splitting up to $125 \mathrm{meV}$ is evident in the defect states, making $\mathrm{PtTe}_{2}$ a strong candidate for spintronic applications. The DoS for each of the plots in Fig. 5 and Supplementary Fig. 9 is shown in Supplementary Fig. 10 allowing direct comparison of the effects of GGA-1/2, as well as SOC, on the DoS of the defective ML PtTe 2 . The influence of defect density on the band structure of $\mathrm{ML} \mathrm{PtTe} 2$ is considered in Supplementary Fig. 11.

\section{DFT-obtained STM of point defects in $\mathrm{PtTe}_{2}$}

To help understand the experimental observations, we systematically explored a variety of defects within a $\mathrm{ML}$ or $\mathrm{BL} \mathrm{PtTe} \mathrm{P}_{2}$ in our ab-initio calculations.

To obtain DFT-based STM images for the defective structures, we considered $\mathrm{ML}$ or $\mathrm{BL}$ slabs to reduce the calculation time, since thicker slabs are extremely computationally demanding. However, in order to ensure that this approach would not diminish the accuracy of the DFT-STM images, we performed the exact same calculations for a Te single-point vacancy in $\mathrm{ML}, \mathrm{BL}$, and trilayer $\mathrm{PtTe}_{2}$ slabs, and observed that there was no difference between the resulting DFT-STM images. This was expected as the images are obtained at a plane just above the top surface of the slab. Critically, as indicated previously in Fig. 4, $\mathrm{PtTe}_{2}$ undergoes a semiconducting-to-semimetallic transition as the film thickness increases. Thus, to be able to consistently compare the DoS with the selected experimental $d / / d V$ spectra (Fig. 3c, g) from the 50- to

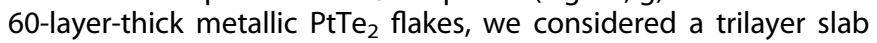
for defect type $C$, which will be discussed later.

Figure 6 shows STM images of fully relaxed DFT-predicted atomic structures of the defect types A-E as defined and listed in Table 1, allowing us to identify the nature of the defects most likely responsible for the features observed in the experimental STM images. As with the experimental data, both positive and negative biases are presented to aid identification. Images under positive bias are presented in the top row, and those at negative bias are shown in the middle row of Fig. 6. Associated top and side views of these relaxed structures are depicted in the bottom row of this figure. Additional DFT-STM images for some other types of defects are shown in Supplementary Figs 12 and 13.

A single chalcogen (here Te) vacancy is a common defect in $\mathrm{TMDs}^{36,50}$, as our formation energy calculation confirms (discussed later in the "Defect formation energy" section). Hence, we first studied the type A defect, whose optimized structure is shown in Fig. 6a. This type of defect is generated by removing a single Te atom from the surface of a ML of $\mathrm{PtTe}_{2}$. Since there are two equivalent surfaces of $\mathrm{ML} \mathrm{PtTe}{ }_{2}$, these two different surfaces are labeled as top and bottom in Table 1. The DFT-STM images based on the calculated structures show a single-site depression at the
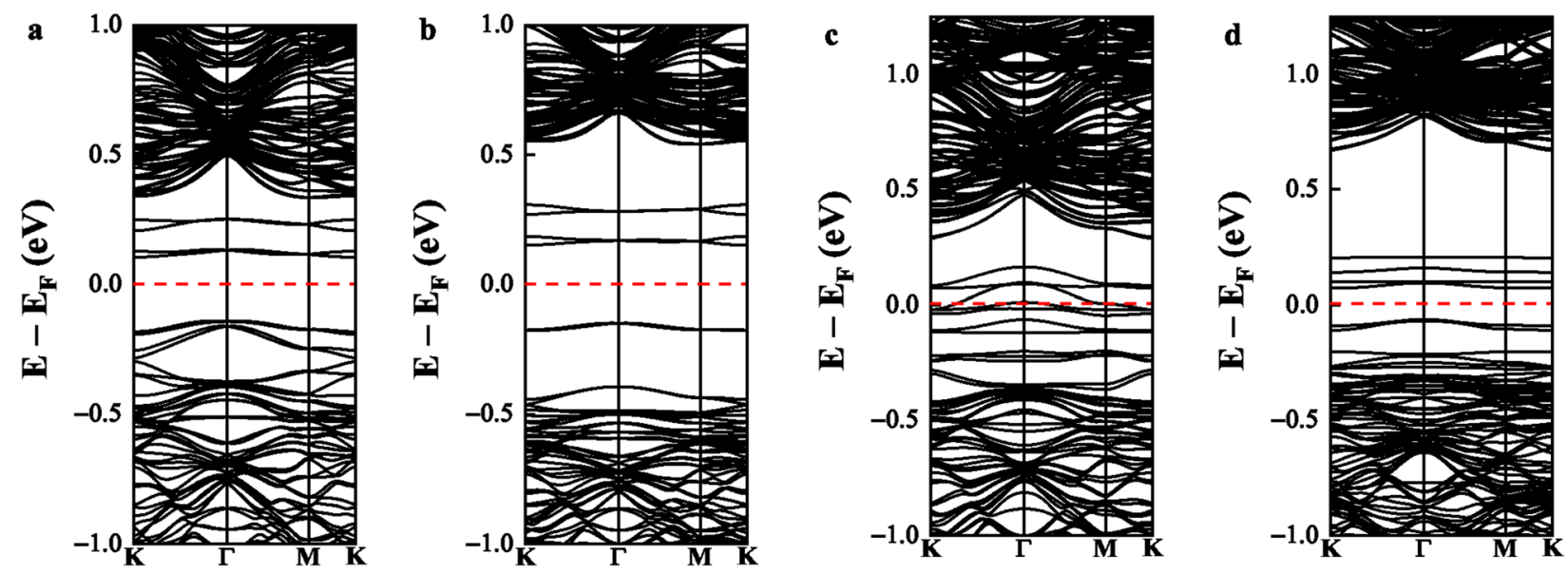

Fig. 5 Influence of vacancies on PtTe $\mathbf{2}_{2}$ band structure. Band structure of ML PtTe 2 with a single Te vacancy (a, b) and single Pt vacancy (c, d) obtained from GGA + SOC and GGA-1/2 + SOC approaches. Energies are referenced to the Fermi energy (red dashed line). 

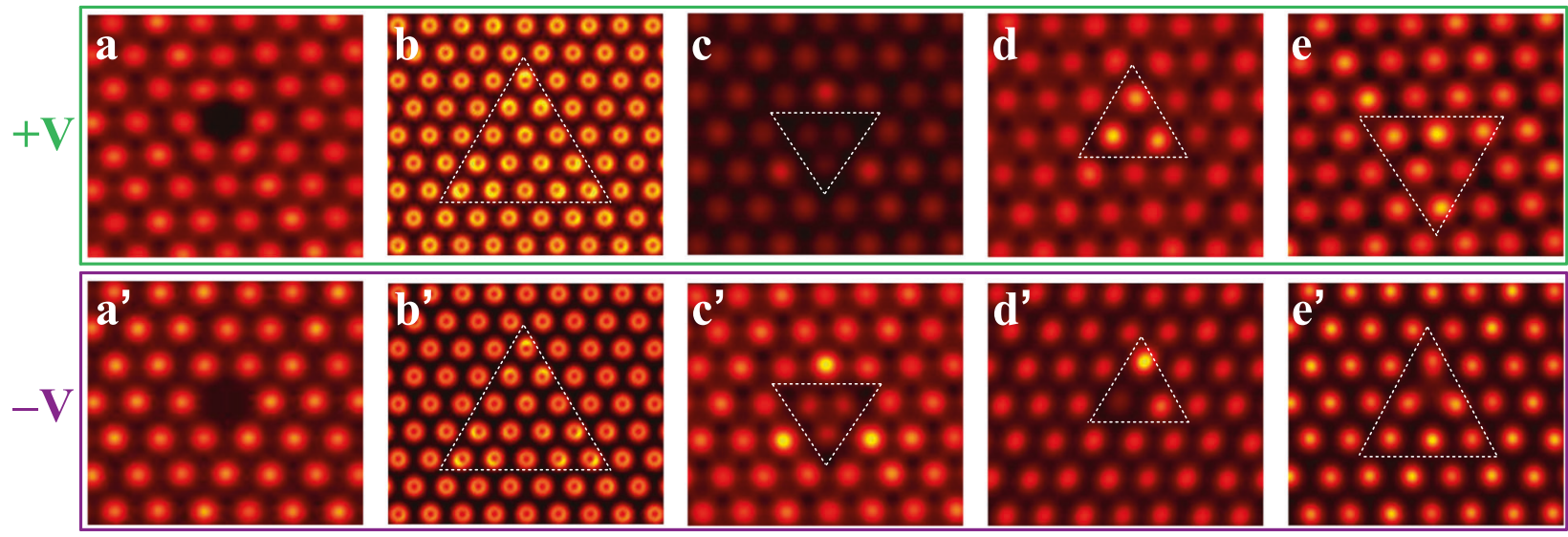
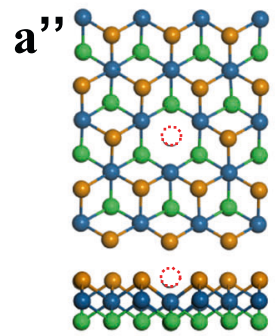
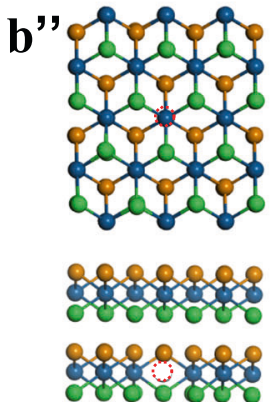
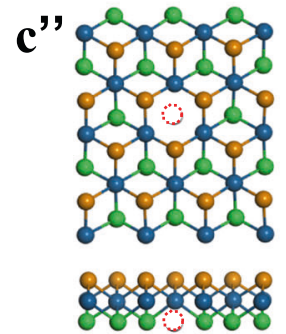

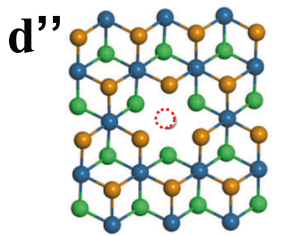

8880888
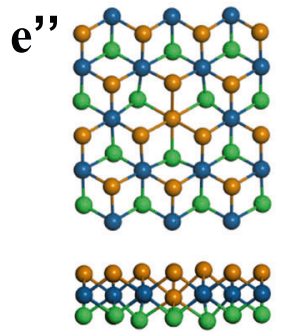

Fig. 6 DFT-STM images of the five types of defects A-E identified. Images (a-e) at positive bias, and images (a'-e') at negative bias conditions. Figures $\left(\mathbf{a}^{\prime \prime}-\mathbf{e}^{\prime \prime}\right)$ the top view and side view of the associated relaxed structures, illustrating the defect in each structure. Red dashed circle represents a vacancy.

surface at both positive and negative biases, and fit quite well with the experimental images shown in Fig. 2a. The effect of this defect type on the band structure of $\mathrm{ML} \mathrm{PtTe} 2$ is shown in Fig. $5 \mathrm{~b}$.

Figure $6 \mathrm{~b}$ shows the DFT-STM images of type $B$ defects where the Pt vacancy in the second layer of BL PtTe 2 leads to a $4 \times 4$ triangular protrusion at both negative and positive biases, which is in good agreement with the experimental STM image. Similar to type $A$, the type $B$ defect DFT-STM images do not show much contrast between positive and negative bias voltages. The band structure of $\mathrm{BL} \mathrm{PtTe}_{2}$ with a $\mathrm{Pt}$ vacancy is presented in Supplementary Fig. 14a. The projected DoS (PDoS) of Pt atoms next to and away from the vacancy is compared in Supplementary Fig. 14b.

For defects of type $C$, a distinct feature has been observed for both positive and negative biases. As indicated by white dotted line in Fig. $6 c$, a $2 \times 2$ triangular depression is formed where outside of middle of each side a bright protrusion spot is clearly visible. These features fit very well with the experimental images shown in Fig. 2c. Notably, a clear contrast between positive and negative bias voltages has been observed for this type of defect. It is worth mentioning that type $A$ and type $C$ defects have the same impact on the band structure of $\mathrm{ML} \mathrm{PtTe}_{2}$.

Defects of type $D$ show $1 \times 1$ triangular protrusions at both positive and negative bias voltages, although the protrusions are much more apparent at positive bias voltage (Fig. 6d). For this defect type, the Pt vacancy, which exists in $\mathrm{ML} \mathrm{PtTe}_{2}$, can build a high acceptor density at the three neighboring Te sites creating the three protrusions at positive bias forming a triangle. This analysis is further supported by the depression created at the position of the Pt vacancy at negative bias voltage (see Fig. $5 \mathrm{~d}$ for the band structure of $\mathrm{ML} \mathrm{PtTe} \mathrm{T}_{2}$ with a Pt vacancy). The observation of slight asymmetry in the images could be related to the asymmetric spatial distribution of charge densities to accommodate the vacancy-induced perturbation of the neutral system, as shown in the Supplementary Fig. 15.
The DFT-STM images for type $E$ defects agree with the experimental STM images. Features such as a $3 \times 3$ triangular protrusion (depression) at negative (positive) bias are observed, but reversed, which is consistent with the experimental images (see Fig. 2e). The impact of this defect type on the band structure of $\mathrm{ML} \mathrm{PtTe} e_{2}$ is presented in Supplementary Fig. 16. This is compared with another possible antisite defect (Pt replacing $\mathrm{Te}$, type $\left.E^{\prime}\right)$, which is energetically much less favorable, as will be discussed in the next section.

As previously noted, due to computationally expensive calculations, we considered ML or BL PtTe 2 to generate the DFT-based STM images of the defects. However, to have fair comparison between calculated DoS and measured $d / / d V$, we performed the atomic-scale simulations on trilayer $\mathrm{PtTe}_{2}$ (without bandgap) in order to calculate the DoS for a Te vacancy (defect type C). The PDoS at Pt and Te atoms near and away from vacancy type $C$ is presented in Supplementary Fig. 17 showing that both Pt and Te atoms next to the Te vacancy create more states in the valence band compared to the conduction band. This result is consistent with the experimental $d / / d V$ data shown in Fig. 3. There is also a good agreement between our simulations and experiments in the conduction band, where a dip is evident after a peak in the DoS. In addition, the PDoS near and away from defect type $E$ is presented in Supplementary Fig. 18.

\section{Defect formation energy}

The formation of different atomic defects depends on the material growth dynamics ${ }^{51}$, which is beyond the scope of this work. Here, we provide a qualitative explanation based on our DFT calculations, to reveal the stability of different defect types, by calculating the formation energies of all the defect types we considered in this work. The formation energies of a Te vacancy and a Pt 


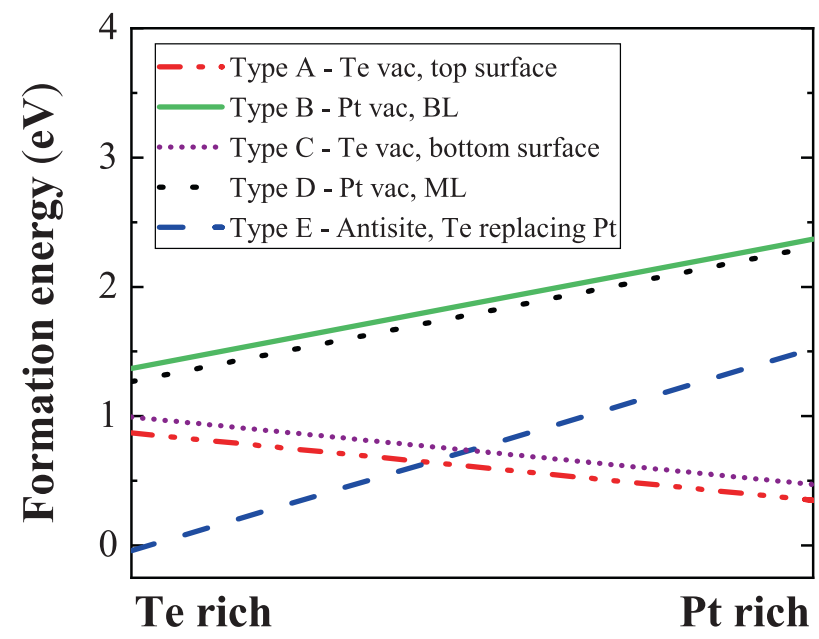

Fig. 7 DFT-calculated formation energies for selected defect types in $\mathbf{P t T e}_{\mathbf{2}}$. Te vacancy has the lowest formation energy. The same plot including some additional less-probable types of defects is shown in Supplementary Fig. 19.

vacancy were calculated using

$E_{f}=E_{\text {tot }}($ defect $)+\sum_{i} N \mu_{i}-E_{\text {tot }}($ pristine $)$

where $E_{\text {tot }}\left(\right.$ defect) and $E_{\text {tot }}$ (pristine) are the total energies of the defective and perfect supercell, respectively. $\mu, i$, and $N$ are the chemical potential, vacancy type, and the number of vacancies, respectively. Chemical potential is related to the growth conditions. In Te-rich conditions, $\mu_{\mathrm{Te}}$ is calculated from the energy of Te in the bulk phase, while for Pt-rich conditions it is derived from $\mu_{\mathrm{Te}}=1 / 2 \times\left(\mu_{\mathrm{PtTe} 2}-\mu_{\mathrm{Pt}}\right)$, where $\mu_{\mathrm{Pt}}$ is the energy of Pt in the bulk phase. Similarly, for a Pt vacancy, $\mu_{\mathrm{Pt}}$ is related to a Pt-rich environment, and for Te-rich conditions, the chemical potential of $\mathrm{Pt}$ is calculated using $\mu_{\mathrm{Pt}}=\mu_{\mathrm{PtTe} 2}-2 \times \mu_{\mathrm{Te}}$. The formation energy $\left(E_{f}\right)$ of the point defects is calculated and plotted in Fig. 7. Our calculated formation energies are consistent with the densities of defects reported in Table 1. For instance, the formation energy of a single Te vacancy is well below the formation energy of a $\mathrm{Pt}$ vacancy in both ML and BL structures. This is in agreement with the higher density of Te vacancy defects observed compared to Pt vacancy defects. The DFT-STM images suggest that defect type E arises from an antisite defect, where Te replaces Pt. In addition, Table 1 shows that the density of defect type $C$ is higher than that of defect type E. This agrees with our result that the formation energy of defect type $C$ is noticeably lower than that of defect type $\mathrm{E}$, which suggests the synthesis process is Pt rich.

For the purpose of comparison, we have also considered lessprobable types of defects, e.g. different configurations of double Te vacancy, triple Te vacancy, and Te and Pt vacancies. DFT-STM images of these types of defects are shown in Supplementary Figs. 12 and 13; formation energies are also compared in Supplementary Fig. 19. As can be seen, for such less-probable defects to occur, higher formation energies compared to the other defect types are required.

In summary, we studied point defects in $\mathrm{PtTe}_{2}$ thin films, which were synthesized through the reaction of solid-phase precursor films, through a combination of experimental STM/STS and DFT simulations. We imaged and identified five different types of point defects. The formation energies for these defects were calculated and found to be in agreement with the density of these defects observed experimentally by STM. Both STS and DFT showed that the defects influence the electronic structure of $\mathrm{PtTe}_{2}$. DFT indicates that $\mathrm{PtTe}_{2}$ is semimetallic for thicknesses greater than $\mathrm{ML}$, confirmed by experimental observation. Moreover, $\mathrm{PtTe}_{2}$ is a semiconductor for the ML case and defects would locally change the bandgap. These findings will play an important role in understanding the electrical properties of $\mathrm{PtTe}_{2}$, the prominent point defects in the structure and how the point defects influence the local electronic structure. These results will be critical for optimizing the synthesis of $\mathrm{PtTe}_{2}$ and tailoring its properties for electronic and spintronic devices through defect engineering.

\section{METHODS}

Experimental details

$\mathrm{PtTe}_{2}$ samples were synthesized through the reaction of solid-phase precursor films. First, a Pt film with $10 \mathrm{~nm}$ thickness was deposited on a substrate by Ar sputtering, with Te (in excess) subsequently electrodeposited upon the Pt film. The sample was then annealed in an inert atmosphere at $450^{\circ} \mathrm{C}$ for $90 \mathrm{~min}$ to form $\mathrm{PtTe}_{2}$. The substrate used was a $\mathrm{SiO}_{2} / \mathrm{Si}$ wafer with a conductive pyrolytic carbon layer on top ${ }^{52}$. The synthesis procedure is described in detail elsewhere ${ }^{11}$.

A commercial low-temperature STM from Createc was used to examine the $\mathrm{PtTe}_{2}$ samples. The STM and STS experiments were performed under UHV with a base pressure of $3 \times 10^{-11} \mathrm{mbar}$. STM images were obtained at a temperature of $77 \mathrm{~K}$ in CCM. Before and after grid STS measurements, the surface positions were checked by STM imaging to confirm the position had remained fixed during the time required to acquire the STS data. The preparation chamber of the STM is equipped with a heating stage for sample annealing. Single-crystalline tungsten tips with a (001) orientation, which were electrochemically etched in $\mathrm{NaOH}$ solution, were utilized. The tunneling bias was applied to the sample. It should be noted that no drift corrections were applied to any STM images shown here. For surface preparation, the sample was annealed up to $430^{\circ} \mathrm{C}$ for $3 \mathrm{~h}$ in UHV, with the temperature measured by the system's thermocouple.

XPS spectra of the Pt $4 f$ and Te $3 d$ core levels were measured by a monochromated Omicron MultiProbe XPS system with an aluminum X-ray radiation source $(1486.7 \mathrm{eV})$. The instrumental resolution of the system is $0.6 \mathrm{eV}$ and a base pressure $5 \times 10^{-11} \mathrm{mbar}$.

A WITec Alpha $300 \mathrm{R}$ with a $532 \mathrm{~nm}$ excitation laser was utilized to collect the Raman spectrum shown in this work. The Raman measurement was obtained with a laser power of $\sim 200 \mu \mathrm{W}$ and using a spectral grating with 1800 lines $/ \mathrm{mm}$ and a $100 \times$ objective lens (N.A. $=0.95$ ). The spectrum shown is an average of a line scan with 20 points, each point having an integration time of $3 \mathrm{~s}$.

XRD analysis was obtained using Bruker D8 Discover equipment with a monochromated $\mathrm{Cu} \mathrm{K}$-alpha source.

SEM images were acquired using a Karl Zeiss Supra microscope operating at an accelerating voltage of $3 \mathrm{kV}$ with a $30 \mu \mathrm{m}$ aperture and a working distance of $\sim 3-4 \mathrm{~mm}$.

\section{Computational details}

First-principle electronic structure calculations were performed based on fully relativistic DFT within the GGA as implemented in QuantumATK ${ }^{53}$. Norm-conserving pseudopotentials for the exchange correlation potential were considered. OpenMx numerical atomic orbital basis sets of $s 2 p 2 d 2 f 1$ were considered for both $\mathrm{Pt}$ and $\mathrm{Te}$ atoms $\mathrm{s}^{54}$. Brillouin-zone integrations were performed over a grid of $k$ points with a density of $\sim 9 \times k$ points per angstrom in Monkhorst-Pack scheme ${ }^{55}$, and the real-space quantities were calculated with an energy cutoff of 250 Rydberg. The localized basis functions allow the consideration of larger supercell dimensions and hence lower vacancy density. Periodic boundary conditions were applied to create simulation supercells, and to avoid any interaction between the periodic images of the neighboring slabs, the cell dimension along the film growth axis was chosen to be $>2 \mathrm{~nm}$. The geometries were fully relaxed until the force acting on each atom was $<0.01 \mathrm{eV} / \AA \AA$. The structure consisted of a single defect in a $6 \times 6 \times 1$ supercell corresponding to a defect density of $2.3 \times 10^{13} \mathrm{~cm}^{-2}$. In the calculation of point defects, an atom from the lattice was replaced by an "empty" atom, which represents a basis set of orbitals with no electron population.

The effect of SOC was considered in the $\mathrm{PtTe}_{2}$ structures due to the strong effect of $5 d$ orbitals in the transition metal atoms. To improve the structural and electronic properties description of this layered material obtained by DFT calculations, van-der-Waals (vdW) interactions were incorporated in our calculations by adding a nonlocal vdW term to the local and semi-local exchange correlation functionals through Grimme's dispersion correction. It is known that the quasiparticle bandgap is underestimated in standard DFT calculations and approximate 
quasiparticle corrections, as implemented in the GGA-1/2 method, have been found to greatly improve the estimation of bandgap energy ${ }^{56,57}$. In the GGA-1/2 method, an atomic self-energy potential is defined to cancel the electron-hole self-interaction energy. This potential is defined as the difference between the potential of the neutral atom and that of a charged ion resulting from the removal of a fraction of its charge. The GGA-1/2 method, which can provide a better description of the defect levels ${ }^{58}$, was also employed in our calculations.

\section{DATA AVAILABILITY}

The data sets generated during and/or analyzed during the current study are available from the corresponding author on reasonable request.

Received: 3 August 2020; Accepted: 11 December 2020;

Published online: 08 January 2021

\section{REFERENCES}

1. Novoselov, K. S. et al. Electric field effect in atomically thin carbon films. Science 306, 666-669 (2004).

2. Chen, Y. et al. Strong correlations and orbital texture in single-layer $1 \mathrm{~T}-\mathrm{TaSe}_{2}$. Nat. Phys. 16, 218-224 (2020).

3. Aristov, V. Y. et al. Layer-by-layer graphene growth on $\beta-S i C / S i(001)$. ACS Nano 13, 526-535 (2019)

4. Rubio-Verdú, C. et al. Visualization of multifractal superconductivity in a twodimensional transition metal dichalcogenide in the weak disorder regime. Nano Lett. 20, 5111-5118 (2020).

5. Goswami, S., Goswami, S. \& Venkatesan, T. An organic approach to low energy memory and brain inspired electronics. Appl. Phys. Rev. 7, 021303 (2020).

6. Butler, S. Z. et al. Progress, challenges, and opportunities in two-dimensional materials beyond graphene. ACS Nano 7, 2898-2926 (2013).

7. Huang, X., Tan, C., Yin, Z. \& Zhang, H. 25th Anniversary article: hybrid nanostructures based on two-dimensional nanomaterials. Adv. Mater. 26, 2185-2204 (2014).

8. Manzeli, S. et al. 2D transition metal dichalcogenides. Nat. Rev. Mater. 2, 17033 (2017).

9. Anasori, B., Lukatskaya, M. \& Gogotsi, Y. 2D metal carbides and nitrides (MXenes) for energy storage. Nat. Rev. Mater. 2, 16098 (2017).

10. Vyas, V. et al. A tunable azine covalent organic framework platform for visible light-induced hydrogen generation. Nat. Commun. 6, 8508 (2015).

11. McManus, J. B. et al. Low-temperature synthesis and electrocatalytic application of large-area PtTe ${ }_{2}$ thin films. Nanotechnology 31, 375601 (2020).

12. Sun, J., Muruganathan, M. \& Mizuta, H. Room temperature detection of individual molecular physisorption using suspended bilayer graphene. Sci. Adv. 2, 1501518 (2016).

13. Kempt, R., Kuc, A. \& Heine, T. Two-dimensional noble-metal chalcogenides and phosphochalcogenides. Angew. Chem. Int. Ed. 59, 9242-9254 (2020).

14. Wang, Q. et al. Electronics and optoelectronics of two-dimensional transition metal dichalcogenides. Nat. Nanotechnol. 7, 699-712 (2012).

15. Lee, Y.-H. et al. Synthesis of large-area $\mathrm{MoS}_{2}$ atomic layers with chemical vapor deposition. Adv. Mater. 24, 2320-2325 (2012).

16. Chih-Pin, L., Guohong, L., Jinhai, M., Li-Min, W. \& Eva, Y. A. Bandgap, mid-gap states, and gating effects in $\mathrm{MoS}_{2}$. Nano Lett. 14, 4628-4633 (2014).

17. Shree, S. et al. High optical quality of $\mathrm{MoS}_{2}$ monolayers grown by chemical vapor deposition. 2D Mater. 7, 015011 (2019).

18. Yan, M. et al. Lorentz-violating type-II Dirac fermions in transition metal dichalcogenide $\mathrm{PtTe}_{2}$. Nat. Commun. 8, 257 (2017).

19. Song, $\mathrm{H}$. et al. Low-temperature eutectic synthesis of $\mathrm{PtTe}_{2}$ with Weak antilocalization and controlled layer thinning. Adv. Funct. Mater. 28, 1803746 (2018)

20. $\mathrm{Hu}, \mathrm{X}$. et al. Infrared nanoimaging of surface plasmons in type-II Dirac semimetal $\mathrm{PtTe}_{2}$ nanoribbons. ACS Nano 14, 6276-6284 (2020).

21. $\mathrm{Xu}, \mathrm{H}$. et al. High spin hall conductivity in large-area type-II Dirac semimetal $\mathrm{PtTe}_{2}$ Adv. Mater. 32, 2000513 (2020).

22. Ma, H. et al. Thickness-tunable synthesis of ultrathin type-II Dirac semimetal PtTe single crystals and their thickness-dependent electronic properties. Nano Lett. 18, 3523-3529 (2018).

23. Politano, A. et al. Tailoring the surface chemical reactivity of transition-metal dichalcogenide PtTe ${ }_{2}$ crystals Adv. Funct. Mater. 28, 1706504 (2018).

24. Rosli, N. F. et al. Layered $\mathrm{PtTe}_{2}$ matches electrocatalytic performance of $\mathrm{Pt} / \mathrm{C}$ for oxygen reduction reaction with significantly lower toxicity. ACS Sustain. Chem. Eng. 6, 7432-7441 (2018)
25. Cheng, P. K. et al. Passively Q-switched Ytterbium-doped fiber laser based on broadband multilayer Platinum Ditelluride $\left(\mathrm{PtTe}_{2}\right)$ saturable absorber. Sci. Rep. 9 10106 (2019).

26. $\mathrm{Xu}, \mathrm{H}$. et al. $\mathrm{PtTe}_{2}$-based type-II Dirac semimetal and its van der Waals heterostructure for sensitive room temperature terahertz photodetection. Small $\mathbf{1 5}$ 1903362 (2019)

27. Schuler, B. et al. Large spin-orbit splitting of deep in-gap defect states of engineered sulfur vacancies in monolayer WS 2 . Phys. Rev. Lett. 123, 076801 (2019).

28. Lin, Z. et al. Defect engineering of two-dimensional transition metal dichalcogenides. 2D Mater. 3, 022002 (2016).

29. Zheng, $\mathrm{H}$. et al. Visualization of point defects in ultrathin layered $1 \mathrm{~T}-\mathrm{PtSe} \mathrm{S}_{2} .2 D$ Mater. 6, 041005 (2019).

30. Barja, S. et al. Identifying substitutional oxygen as a prolific point defect in monolayer transition metal dichalcogenides. Nat. Commun. 10, 3382 (2019).

31. Edelberg, D. et al. Approaching the intrinsic limit in transition metal diselenides via point defect control. Nano Lett. 19, 4371-4379 (2019).

32. Zhang, S. et al. Defect structure of localized excitons in a $\mathrm{WSe}_{2}$ monolayer. Phys. Rev. B 119, 046101 (2017).

33. Cochrane, K. A. et al. Intentional carbon doping reveals $\mathrm{CH}$ as an abundant charged impurity in nominally undoped synthetic $\mathrm{WS}_{2}$ and $\mathrm{WSe}_{2} .2 D$ Mater. 7 , 031003 (2020).

34. Denga, K. et al. Crossover from 2D metal to 3D Dirac semimetal in metallic PtTe films with local Rashba effect. Sci. Bull. 64, 1044-1048 (2019).

35. Komsa, H. P., Kurasch, S., Lehtinen, O., Kaiser, U. \& Krasheninnikov, A. V. From point to extended defects in two-dimensional $\mathrm{MoS}_{2}$ : evolution of atomic structure under electron irradiation. Phys. Rev. B 88, 035301 (2013).

36. Hong, J. et al. Exploring atomic defects in molybdenum disulphide monolayers. Nat. Commun. 6, 6293 (2015).

37. Wang, S., Robertson, A. \& Warner, J. H. Atomic structure of defects and dopants in 2D layered transition metal dichalcogenides. Chem. Soc. Rev. 47, 6764-6794 (2018).

38. Zhussupbekov, K. et al. Electronic and structural characterisation of polycrystalline platinum disulfide thin films. RSC Adv. 10, 42001-42007 (2020).

39. Caffrey, D. et al. Crystallographic characterisation of ultra-thin, or amorphous transparent conducting oxides-the case for Raman spectroscopy. Materials 13, 267 (2020).

40. Anemone, G. et al. Experimental determination of surface thermal expansion and electron-phonon coupling constant of 1T-PtTe $2.2 D$ Mater. 7, 025007 (2020).

41. Zhang, K. et al. Experimental evidence for type-II Dirac semimetal in $\mathrm{PtSe}_{2}$. Phys. Rev. B 96, 125102 (2017).

42. Frolov, A. S. et al. Atomic and electronic structure of a multidomain GeTe crystal. ACS Nano 14, 16576-16589 (2020).

43. Zhussupbekov, K. et al. Oxidation of $\mathrm{Nb}(110)$ : atomic structure of the $\mathrm{NbO}$ layer and its influence on further oxidation. Sci. Rep. 10, 3794 (2020).

44. Hildebrand, B. et al. Doping nature of native defects in $1 \mathrm{~T}-\mathrm{TiSe}_{2}$. Phys. Rev. Let. 112, 197001 (2014).

45. Ansari, L., Fagas, G., Colinge, J.-P. \& Greer, J. C. A proposed confinement modulated gap nanowire transistor based on a metal (Tin). Nano Lett. 12, 2222-2227 (2012).

46. Gity, F. et al. Reinventing solid state electronics: harnessing quantum confinement in bismuth thin films. Appl. Phys. Lett. 110, 093111 (2017).

47. Ansari, L., Fagas, G., Gity, F. \& Greer, J. C. A sub k ${ }_{B} T / q$ semimetal nanowire field effect transistor. Appl. Phys. Lett. 109, 063108 (2016).

48. Ansari, L. et al. Quantum confinement-induced semimetal-to-semiconductor evolution in large-area ultra-thin $\mathrm{PtSe}_{2}$ films grown at $400^{\circ} \mathrm{C}$. NPJ $2 \mathrm{D}$ Mater. Appl. 3, 33 (2019).

49. Heyd, J. \& Scuseria, G. E. Hybrid functionals based on a screened Coulomb potential. J. Chem. Phys. 118, 8207 (2003)

50. Vancsó, P. et al. The intrinsic defect structure of exfoliated $\mathrm{MoS}_{2}$ single layers revealed by Scanning Tunneling Microscopy. Sci. Rep. 6, 29726 (2016).

51. Gao, J. et al. Structure, stability, and kinetics of vacancy defects in monolayer $\mathrm{PtSe}_{2}$ : a first-principles study. ACS Omega 2, 8640-8648 (2017).

52. McEvoy, N. et al. Synthesis and analysis of thin conducting pyrolytic carbon films. Carbon 50, 1216-1226 (2012).

53. Smidstrup, S. et al. QuantumATK: an integrated platform of electronic and atomic-scale modelling tools. J. Phys. Condens. Matter 32, 015901 (2019).

54. Ozaki, T. Variationally optimized atomic orbitals for large-scale electronic structures. Phys. Rev. B 67, 155108 (2003).

55. Monkhorst, H. J. \& Pack, J. D. Special points for Brillouin-zone integrations. Phys. Rev. B 13, 5188 (1976).

56. Ferreira, L. G., Marques, M. \& Teles, L. K. Approximation to density functional theory for the calculation of band gaps of semiconductors. Phys. Rev. B 78 125116 (2008). 
57. Ferreira, L. G., Marques, M. \& Teles, L. K. Slater half-occupation technique revisited: the LDA-1/2 and GGA-1/2 approaches for atomic ionization energies and band gaps in semiconductors. AIP Adv. 1, 032119 (2011).

58. Lucatto, B. et al. General procedure for the calculation of accurate defect excitation energies from DFT-1/2 band structures: The case of the NV center in diamond. Phys. Rev. B 96, 075145 (2017).

\section{ACKNOWLEDGEMENTS}

I.V.S. acknowledges the support of SFI PI_12/IA/1264 and IRC LA (IRCLA/2019/171), G. S.D., J.B.M.M., C.Ó.C., P.K.H., and F.G. acknowledge the support of SFI under PI_15/IA/ 3131. N.M. acknowledges support from SFI through $15 /$ SIRG/3329 and N.M, F.G., and P.K.H. acknowledge SFI 12/RC/2278_P2. K.Z. and A.Z. acknowledge the support of Government of Kazakhstan under the Bolashak program. SFI/HEA Irish Center for High-End Computing (ICHEC) is acknowledged for the provision of computational facilities and support. L.A. would also like to acknowledge funding from Irish Research Council (IRC) through award GOIPD/2018/653. G.S.D. acknowledges the European Commission under the project Graphene Flagship (881603) and the German Ministry of Education and Research (BMBF) under the project NobleNEMS (16ES1121).

\section{AUTHOR CONTRIBUTIONS}

K.Z. and C.Ó.C. performed STM/STS measurements, and analyzed data. K.Z., C.Ó.C., and N.M. designed practical experiments. L.A. and F.G. conducted the theoretical calculations and atomic-scale simulations. L.A. and F.G. designed computational experiments. J.B.M. synthesized $\mathrm{PtTe}_{2}$ and performed spectroscopic characterization. A.Z. performed spectroscopic characterization. I.V.S., G.S.D., P.K.H., and N.M. helped with the planning of the experiments and the interpretation of the results. F.G., C.O.C., and N.M. supervised the project. The manuscript was primarily written by K.Z., L.A., F. G., C.Ó.C., and N.M., but involved contributions from all authors. All authors have given approval to the final version of the manuscript.

\section{COMPETING INTERESTS}

The authors declare no competing interests.

\section{ADDITIONAL INFORMATION}

Supplementary information is available for this paper at https://doi.org/10.1038/ s41699-020-00196-8.

Correspondence and requests for materials should be addressed to F.G., C.Ó C. or N.M.

Reprints and permission information is available at http://www.nature.com/ reprints

Publisher's note Springer Nature remains neutral with regard to jurisdictional claims in published maps and institutional affiliations.

(c) Open Access This article is licensed under a Creative Commons adaptation, distribution and reproduction in any medium or format, as long as you give appropriate credit to the original author(s) and the source, provide a link to the Creative Commons license, and indicate if changes were made. The images or other third party material in this article are included in the article's Creative Commons license, unless indicated otherwise in a credit line to the material. If material is not included in the article's Creative Commons license and your intended use is not permitted by statutory regulation or exceeds the permitted use, you will need to obtain permission directly from the copyright holder. To view a copy of this license, visit http://creativecommons. org/licenses/by/4.0/.

(c) The Author(s) 2021 